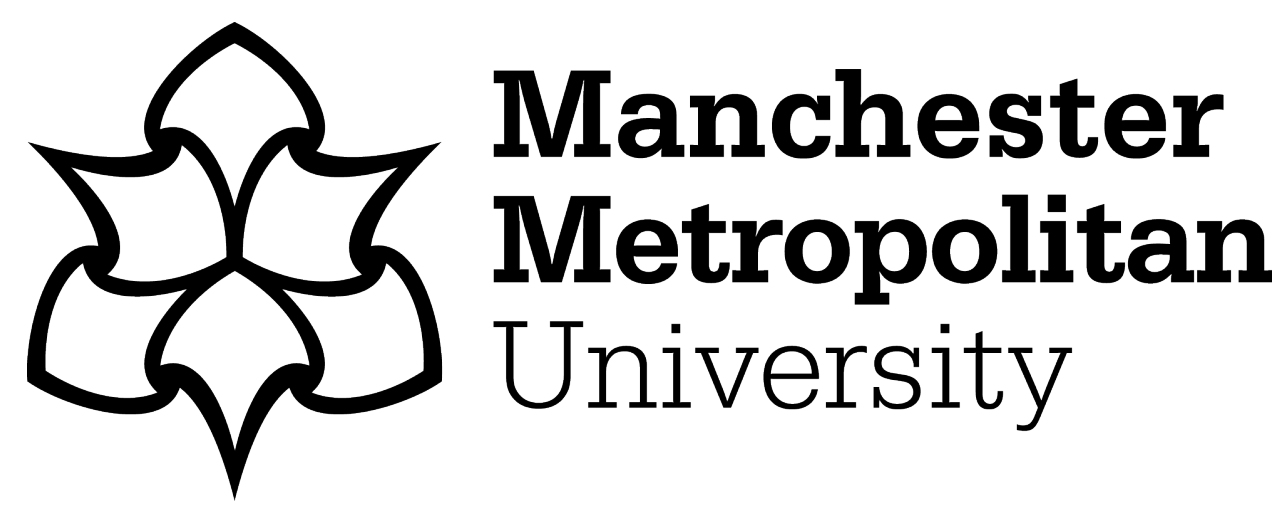

Hoogendoorn, CJ, Gonzalez, JS, Schechter, CB, Flattau, A, Reeves, ND, Boulton, AJM and Vileikyte, $L$ (2021) Correlates of self-rated health in people with diabetic peripheral neuropathy: a longitudinal study. Diabetic medicine : a journal of the British Diabetic Association, 38 (5). e14383. ISSN 0742-3071

Downloaded from: https://e-space.mmu.ac.uk/626424/

Version: Accepted Version

Publisher: Wiley

DOI: https://doi.org/10.1111/dme.14383

Please cite the published version 


\section{Correlates of Self-Rated Health in Patients with Diabetic Peripheral Neuropathy: A Longitudinal Study}

\section{Running title: Diabetic Peripheral Neuropathy and Self-Rated Health}

Claire J. Hoogendoorn, $\mathrm{PhD}$ 1, Jeffrey S. Gonzalez, $\mathrm{PhD}_{1,2,3}$, Clyde B Schechter, MD4, Anna Flattau, MD4, Neil D. Reeves, PhD5, Andrew J.M. Boulton, MD6,7 \& Loretta Vileikyte, MD, $\mathrm{PhD} 6,7$

1Ferkauf Graduate School of Psychology, Yeshiva University, Bronx, NY, USA; 2Departments of Medicine (Endocrinology) and Epidemiology \& Population Health, Albert Einstein College of Medicine, Bronx, NY, USA; 3 The Fleischer Institute for Diabetes and Metabolism, Albert Einstein College of Medicine, Bronx, NY, USA; 4Department of Family \& Social Medicine, Albert Einstein College of Medicine, Bronx, NY, USA; 5 Research Centre for Musculoskeletal Science and Sports Medicine, Department of Life Sciences, Manchester Metropolitan University, Manchester, UK; 6 Division of Diabetes, Endocrinology and Gastroenterology, Faculty of Biology, Medicine and Health, The University of Manchester, Manchester, UK; 7Diabetes Research Institute, Miller School of Medicine, University of Miami, Miami, Fl, USA;

Grant Support: This study was supported by Diabetes UK (PI: Dr. Vileikyte; RD00-0002009) and the American Diabetes Association (PI: Dr. Rubin; 1-00-CR-11). The authors would also like to acknowledge the support of the Einstein-Mount Sinai Diabetes Research Center (P30 DK020541) and the New York Regional Center for Diabetes Translation Research (P30DK111022). Dr. Hoogendoorn is supported by the Drs. David and Jane Willner Bloomgarden Family Fellowship Fund. Dr. Gonzalez is supported by the following grants from the National Institutes of Health (NIH): R18 DK098742, R01 DK104845, R01DK121298, R01 DK121896.

\section{Correspondence to:}

Claire J. Hoogendoorn

Ferkauf Graduate School of Psychology

1165 Morris Park Avenue, Rousso Building

Bronx, NY 10461

Phone: 646-592-4569

Fax: 718-430-3960

Email: claire.hoogendoorn@yu.edu

Text word count: 2,996

Abstract word count: 249

Number of Tables: 3, Number of Figures: 0

Conflicts of interest: None of the authors have conflicts of interests to disclose. 


\section{Bulleted novelty statement:}

- Self-rated health (SRH) is a robust independent predictor of morbidity and mortality in various populations, including persons with diabetes. However, the correlates of SRH among people with complications of diabetes remain understudied.

- Objectively assessed diabetic peripheral neuropathy (DPN) severity was associated with lower SRH, and this relationship appeared to be mediated by associated increases in DPN-related symptoms, DPN-specific limitations in daily activities, and depression symptoms.

- The finding that SRH is linked to important health indicators among those with DPN support the growing interest to systematically assess SRH and other patient-reported outcomes in diabetes research and care. 
Acknowledgments: C.J.H. contributed the study concept and wrote and revised the manuscript. J.S.G. and L.V. contributed to the study concept and revisions of the manuscript. C.B.S. contributed to data analyses and revisions of the manuscript. A.F., N.D.R. and A.J.M.B contributed to revisions of the manuscript. This study was supported by Diabetes UK (PI: Dr. Vileikyte; RD00-0002009) and the American Diabetes Association (PI: Dr. Rubin; 1-00-CR-11). This work is also supported by the Einstein-Mount Sinai Diabetes Research Center (P30 DK020541) and the New York Regional Center for Diabetes Translation Research (P30 DK111022). Dr. Hoogendoorn is supported by the Drs. David and Jane Willner Bloomgarden Family Fellowship Fund. Dr. Gonzalez is also supported by NIH grants: R18 DK098742, R01 DK104845, R01DK121298, R01 DK121896. 


\begin{abstract}
OBJECTIVE: Self-rated health (SRH) is a robust predictor of morbidity and mortality in various populations, including persons with diabetes. The aim of this longitudinal investigation was to examine correlates of SRH in adults with diabetic peripheral neuropathy (DPN).

RESEARCH DESIGN AND METHODS: Participants ( $\mathrm{n}=295$; age M(SD)=61.5 \pm 10.7 years; $70 \%$ male; $73 \%$ type 2 diabetes) provided SRH (SF-12) at baseline and 18-months, and completed the Neuropathy and Foot Ulcer-Specific Quality of Life measure assessing DPNsymptoms of pain, unsteadiness and reduced sensation in feet, DPN-specific limitations in daily activities, and DPN-specific distress. The Hospital Anxiety and Depression Scale assessed symptoms of depression. DPN severity was assessed with the Neuropathy Disability Score and the Vibration Perception Threshold.
\end{abstract}

RESULTS: At baseline, greater neuropathy severity was associated with worse SRH when controlling for covariates (coef.[95\%CI]=-.06[-.11--.01]). This relationship appeared to be mediated by symptoms of reduced feeling (-.11[-.20--.01]), increased pain (-.17[-..31--.02]) and increased unsteadiness (-.16[-.28--.04]), greater limitations in daily activities (-.13[-.22--.04]) and more depression symptoms (-.09[-.12--.05]). SRH was reasonably stable over 18 months when adjusting for predictors $(I C C=0.59[0.51-0.67])$. At 18-months, unsteadiness (-.07[-.19--.01]), limitations in daily activities (-.08[-.14--.02]), depression symptoms (-.06[-.09--.04]) and distress ($.10[-.20--.001])$ showed independent associations with worse SRH.

CONCLUSIONS: Results identify independent correlates of SRH among adults with diabetic peripheral neuropathy and suggest that, if these effects represent causal relationships, neuropathy severity affects patients' perceptions of their health through the experience of DPN-related symptoms, limitations in daily activities, and depression symptoms and DPN-related emotional distress.

Keywords: diabetic peripheral neuropathy, self-rated health, unsteadiness, depression symptoms, diabetes-related distress. 
Patient-reported outcomes (PROs) are increasingly important in clinical practice and clinical trials to capture valuable information about health status and burdens of treatment, not otherwise reflected in objective or biological measures. One central PRO is self-rated health (SRH), which refers to a person's perceptions of their general health and incorporates social, psychological and biological aspects of the self (1). Despite robust controls for potential confounding variables, responses to various single-item measures of SRH consistently and robustly predict morbidity and mortality (2), including among individuals with diabetes (3). SRH has been shown to predict diabetic foot ulcerations (DFUs) and amputations over 2.4 years of follow-up among individuals with type 2 diabetes after adjustment for established risk factors and clinical history (4). While complications of diabetes such as diabetic peripheral neuropathy (DPN) show a consistent relationship with poorer SRH (e.g., 5), the correlates of SRH among people with complications of diabetes remain understudied.

Diabetic peripheral neuropathy is the most prevalent complication of diabetes, affecting up to $50 \%$ of individuals with diabetes (6) and leading to severe morbidity and extremely high health care costs (7). Patients experience impaired quality of life (QOL) due to neuropathic pain, loss of sensation leading to poor balance, falls, foot deformities, and high rates of ulceration and amputation (8). DPN is a source of significant emotional distress, with DPN symptoms and associated limitations in functioning accounting for nearly half of the variance in depression symptom scores $(9,10)$. DPN-related unsteadiness is one of the strongest predictors of depression symptoms in this patient population $(9,10)$ and could be an important influence on nonadherence to offloading interventions (11). An earlier cross-sectional study linked DPN symptoms of numbness and loss of sensation with lower overall SRH (5), but the whole range of DPN symptoms was not evaluated and clinical indicators of DPN severity were not available. 
The current study explores independent correlates of patients' perceptions SRH over time among individuals with DPN. We hypothesized that increased DPN severity would be associated with lower SRH, independent of demographic and other disease-related characteristics. We also hypothesized that DPN severity would be associated with SRH through the experience of DPNrelated symptoms and limitations in daily activities. Finally, we expected that factors would show consistent associations with SRH over time. A better understanding of correlates of SRH in patients with DPN may shed light on the factors that could influence perceptions of health among these patients and would provide evidence for the validity and clinical significance of SRH as a PRO among individuals with DPN.

\section{RESEARCH DESIGN AND METHODS}

\section{Participants and Procedures}

A total of 295 individuals with Type 1 or Type 2 diabetes and moderate-to-severe DPN who provided SRH at baseline and 18-months were included in the current analyses. These individuals were recruited from specialist diabetes centers in the UK (Manchester) and USA (Baltimore, MD and State College, PA) for participation in an 18-month study examining the psychological determinants of foot self-care adherence and foot ulceration; further details of this sample have been previously described $(9,10,12)$. Two tests were used to establish the presence of DPN in accordance with diagnostic guidelines (6): the neuropathy disability score (NDS) and the vibration perception threshold (VPT), which are further described in the 'Measures' section. Patients were included if VPT $\geq 25$ volts and NDS $\geq 3$. Those with milder DPN severity and those who had significant peripheral vascular disease $(<1$ palpable pulse per foot and/or history of bypass surgery), history of major amputation (> a single digit), advanced diabetes 
complications (e.g., end-stage renal disease), other severe medical conditions (e.g., stroke), or insufficient English comprehension were excluded from participation.

The study was approved by the Central Manchester Research Ethical Review Committee and the Institutional Review Boards at the Johns Hopkins University and the Pennsylvania State University, and informed consent was obtained from all participants.

\section{Measures}

Demographic, disease and trait characteristics. Demographic and clinical characteristics assessed included age, sex, education, marital status, type and duration of diabetes, diabetes complications (retinopathy, nephropathy, and cardiovascular disease), and number of comorbid illnesses. Neuroticism was included to adjust for a general predisposition to experience negative affect and was measured by the Big Five Inventory (8 items) (13), which showed good internal consistency in this sample (alpha=.82). Presence of retinopathy and nephropathy, and age were reassessed at 18 -months.

DPN Severity. The VPT was assessed at the great toe in both feet in triplicate, using a neurothesiometer (Horwell, Nottingham, UK). The NDS score was derived from examination of pain, vibration, temperature sensation and Achilles reflex. A score of 0 represents a normal nervous system examination and the maximum score was 10 (14). NDS was reassessed at 18 months.

DPN-related Symptoms and Functioning. The NeuroQol, a measure of quality of life developed specifically for individuals with DPN (15), includes neuropathic symptoms of reduced feeling ( 3 items), pain ( 7 items), and unsteadiness ( 3 items), DPN-related limitations in daily activities (3 items), and DPN-specific distress (11 items). Higher scores indicate greater endorsement of symptoms and limitations in functioning. Internal consistency for DPN 
symptoms, DPN-related limitations in daily activities, and DPN-specific distress was good to excellent (alphas $=.86-.90)$. The NeuroQol was administered at both baseline and 18 months .

History of DFUs. Individuals were coded as having a positive history of DFU if they selfreported a prior DFU or had an active DFU determined by a medical examination. A history of DFUs was obtained by asking each subject: "Have you ever had a foot ulcer (an open sore on your foot)?" Those answering in the affirmative were verified by examination of medical records and careful podiatric assessment. A foot ulcer was defined as a full thickness skin break below the malleoli. A history of DFUs was assessed only at baseline.

Emotional Distress. Assessment of DPN-specific distress using the NeuroQol is described above. Depressive symptoms were assessed using the 7-item Hospital Anxiety and Depression Scale (HADS) (16). The HADS was selected as it was designed to reduce confounding of somatic symptoms that commonly occur in the presence of physical illness. Questions included both positive (e.g., I can laugh and see the funny side of things) and negative (e.g., I feel as I am slowed down) endorsement of depression. Questions were coded so that higher scores indicate greater severity of symptoms. Internal consistency was good (alpha $=.84)$. Emotional distress measures were administered at both time points.

Self-Rated Health. SRH was measured by a single item from the SF-12 (17) at baseline and the 18-month follow-up, "In general, would you say your health is excellent, very good, good, fair, or poor?" Responses were rated on a 1-5 scale and were reverse coded so that higher scores indicate better health.

\section{Statistical Analyses}


Descriptive statistics were assessed for all study-related variables. Bivariate associations were examined using Pearson's $r$ for continuous study variables, and t-tests or chi square for categorical study variables.

Six regression models were run to explore the association between hypothesized correlates and SRH assessed at baseline, as follows: 1) control variables (demographic/ disease/ personality characteristics), 2) objective indicators of neuropathy severity (NDS and VPT scores), 3) History of DFUs, 4) DPN-related symptoms, 5) DPN-related limitations in daily activities, and 6) depressive symptoms and DPN-specific distress. These variables were entered in separate steps to test independent associations with SRH in a way that aligned with our hypotheses.

One mixed-effects regression model was run (model 7), with random intercepts at the patient level, to test whether factors were consistently associated with SRH over time. This model was selected as SRH remained relatively stable over time; and it tests the relationship between correlates and SRH at 18-months while accounting for multiple assessments. This model only included variables collected at both baseline and 18-months.

Data were considered to be missing at random, with no demographic or illness differences between those missing and not missing data. There were 9 variables with some missing values, ranged from 1 missing value for the presence of CVD to 9 for the presence of retinopathy. We ran multiple imputation analyses for baseline models to account for missing data. Parameters were estimated within each dataset individually and then combined using Rubin's rules (18). Analyses were conducted using Stata 15.1 (19).

\section{RESULTS}

\section{Sample Characteristics}


Descriptive characteristics of the study sample are presented in Table 1 . The majority of participants were White, male, older adults diagnosed with Type 2 diabetes. In this population with DPN, retinopathy (44.1\%) and cardiovascular disease $(34.9 \%)$ were the most common additional diabetes complications reported. At baseline, $38.3 \%$ of subjects either reported a previous or active DFU. The most commonly endorsed SRH score indicated "Good" health (37.6\%), followed by "Fair" health $(31.2 \%)$. The mean HADS score $(4.93 \pm 3.81)$ was within the "normal" range (21), and the mean DPN-emotional distress score $(2.3 \pm 1.2)$ indicated average distress (20).

Site comparisons (UK vs US) indicated that US participants reported more comorbid disorders, more DFUs, higher VPT scores indicative of more severe DPN, and lower selfreported functioning as assessed by the NeuroQoL.

\section{Summary of Findings}

Baseline Analyses

Results of the six stepped regression models are shown in Table 2. Model 1 examined demographic and disease correlates of baseline SRH; being in the US ( $p=.022)$, younger age $(p=.003)$, presence of CVD ( $p=.001)$, higher number of comorbidities $(p=.027)$ and neuroticism ( $p<.001)$ showed independent associations with lower baseline SRH. When NDS and VPT scores were added to the model (Model 2), NDS scores independently predicted baseline SRH $(p=.033)$. The addition of DFU history to create Model 3, did not support an independent association with baseline SRH. When DPN symptoms assessed by the NeuroQOL were added to create Model 4, reduced feeling ( $p=.032)$, pain $(p=.031)$ and unsteadiness $(p=.010)$ showed independent associations with baseline SRH and attenuated the effect of NDS on baseline SRH. When DPN-specific limitations in daily activities scores from the NeuroQol were added to 
model 5 (Table 2) this also showed an independent association with baseline SRH $(\mathrm{p}<.01)$ and attenuated the independent associations between reduced feeling and pain symptoms with baseline SRH, with only symptoms of unsteadiness continuing to show an independent association with SRH ( $p=.040)$. Finally, when depression and DPN-specific distress were included in model 6, depression symptoms showed an association with baseline SRH $(\mathrm{p}<.001)$ and attenuated the effect of DPN-specific limitations in daily activities and unsteadiness (Table 2, Model 6). In this final model, being in the US ( $p=.045)$, younger age ( $p=.011)$, presence of CVD ( $p=.003)$, a higher number of comorbidities ( $p=.049)$, neuroticism $(p=.049)$, a lower VPT score $(p=.026)$, and depression symptoms $(\mathrm{p}<.01)$ indicated an independent association with lower SRH.

\section{Longitudinal Analyses}

The intra-class correlation between SRH at baseline and 18 months was 0.59 (95\%CI: 0.51-0.67) when accounting for the predictors in the model, suggesting a reasonably good stability of SRH over time. The mixed-effects model indicated that being in the US ( $p=.017$ ), reporting more unsteadiness ( $p=.029)$, a higher level of DPN-specific limitations in daily activities $(p=.015)$, higher levels of depression symptoms $(p<.01)$, and DPN-specific distress ( $p=.048)$ were each independently related to higher SRH at 18 -months when accounting for multiple assessments (Table 3, Model 7).

\section{DISCUSSION}

The goal of this study was to identify correlates of patients' perceptions of their health, and assess these associations longitudinally, among a sample of patients with DPN. Our findings extend work of Klein and colleagues (5) conducted over 30 years ago that demonstrated selfreported neuropathy symptoms were strongly associated with lower SRH among 937 diabetes 
patients. Our work reproduces these findings using objective measures of DPN. This is important because self-reported symptoms of DPN do not consistently correlate with objective measures of DPN severity (20), and because self-reported and objectively measured DPN symptoms can differ in their relationship to patient outcomes (9). If these effects represent causal relationships, results further suggest that neuropathy severity affects patients' perceptions of their health through the experience of DPN-related symptoms, limitations in daily activities, and depression symptoms and DPN-related emotional distress. Results also supported that SRH was reasonably stable over an 18-month period, which may reflect the fact that examined correlates (and complications like DPN) are likely to be relatively stable over an 18-month time frame. While not all measures were assessed at both time points, greater DPN-related unsteadiness, greater DPN-specific limitations in daily activities, and higher depression symptoms consistently showed independent associations with SRH over time.

This study also identified several demographic and disease-related characteristics as independent correlates of lower SRH among persons with DPN. This included living in the US, younger age, presence of CVD, a higher number of comorbidities, and neuroticism. The independent association between living in the US compared to the UK and lower SRH scores, in addition to observed group differences (e.g., higher disease severity and lower functioning among individuals from the US), may reflect cultural differences, or differences in recruitment or access to social services and health care between countries. The independent association observed between younger age and lower SRH may be reflective of an earlier onset of DPN, though previous literature on the relationships between age, age of illness onset, and SRH show mixed findings that may differ by gender $(3,5)$. Future work should continue to examine how these factors relate to SRH and health outcomes over time among individuals with DPN. 
A history of DFUs was not independently associated with patients' perceptions of health when adjusting for demographic factors, comorbid health conditions, and severity of DPN. This adds to findings of a population-based study conducted in Norway that reported an independent relationship between SRH and DFU history when adjusting for demographic factors, comorbid health conditions, lifestyle variables, and illness-related variables such as insulin use, A1C, and diabetes duration (21). This Norwegian study differed from the current study in that they assessed a population-based sample of individuals with diabetes, rather than a sample of individuals diagnosed with DPN. Relatedly, while Iversen and colleagues (21) accounted for variables related to disease severity, they did not control for severity of neuropathy. Additionally, a history of DFUs were assessed only via self-report in the Norwegian study, while the current study verified self-reports by examination of medical records and podiatric assessment. Additional research is needed to reach a clearer answer on the independent association of DFU history and SRH among various diabetes samples.

While we are not able to establish causality, our work shows that physical symptoms, DPN-specific limitations in daily activities and depressive symptoms may be a mechanism by which DPN severity contributes to reduced SRH. This is consistent with previous data showing that physical symptoms and functional status predict SRH among individuals with type 2 diabetes (22). Heightened emotional distress has been posited as a pathway through which DPN symptoms like unsteadiness negatively impact SRH and QOL (23), and previous data from this cohort showed that DPN-specific symptoms and limitations in daily activities were predictive of depression symptom severity cross-sectionally and over time $(9,10)$. The current findings are consistent with a pathway of DPN-severity contributing to SRH ratings through increased symptoms, reduced functioning, and increased emotional distress. 
Previous research suggests that both physical and emotional functioning contribute to SRH. Our finding that depressive symptoms showed the largest predictive power among persons with DPN maps onto an inconsistent literature. Previous research supports that physical symptoms and other health-related measures show the largest predictive power compared to psychological well-being in a population-based study (1) and among individuals with diabetes (24). Yet, other population-based studies show depression to be one of the largest predictors of fair or poor SRH (e.g., 25). It is possible that the relative strengths of relationships between emotional and physical functioning and SRH may differ among different samples or with the inclusion of different measures of physical and emotional functioning.

The current study is limited in that it consists of two discrete (US and UK) samples of mostly White men, which limit generalizability of findings. Further, causal inferences cannot be made from this study: future studies with an experimental design that may manipulate correlates of SRH (e.g., one's level of unsteadiness, emotional distress) would allow for better assessment of the direction of influence underlying the observed associations between patient and illness factors and SRH over time.

It is difficult to make specific clinical recommendations until DPN-related correlates of SRH are further clarified. It may be beneficial for clinicians to be more attentive to DPN symptom severity and particularly unsteadiness, as this symptom is often under-reported and underdiagnosed (20). Recent work has shown that perceived DPN-related unsteadiness is closely associated with gait laboratory assessments of walking and balance impairment, thus individuals with DPN appear to have a good sense of their level of impairment (26). It may be beneficial to assess the impact of experienced symptoms on limitations in daily activities and emotional wellbeing. Once identified, multifaceted interventions that target both biomechanical difficulties and 
emotional distress among patients with DPN hold the potential to improve SRH as well as QOL (27). Future research should test such integrative interventions and continue to model the myriad of aspects of health, well-being an expectations that come together to contribute to the important PRO of SRH among individuals with DPN.

Inclusion of PROs such as SRH in DPN-related clinical care and research promotes a patient-centered focus, and SRH may be particularly useful in that it consists of a single-item question that can be easily incorporated into health services and research. Future research should examine whether perceptions of health predict important medical outcomes such as mortality in this patient population. This would contribute to our understanding of SRH as a risk factor for negative health outcomes as diabetes progresses and patients develop complications.

Additionally, further work is needed to better understand how SRH maps onto other important PROs such as QOL, and the importance of distinguishing health status from QOL has been reviewed (e.g., 28,29). The finding that SRH is linked to multiple important health indicators support the growing interest to systematically assess PROs as primary outcomes in diabetes research. Importantly, centering PROs like SRH in diabetes research and care aligns with American Diabetes Association's recommendations for a patient-centered approach to diabetes care (30). 


\section{References}

1. Singh-Manoux A, Martikainen P, Ferrie J, Zins M, Marmot M, Goldberg M. What does self rated health measure? Results from the British Whitehall II and French Gazel cohort studies. J Epidemiol Community Health 2006;60:364-72.

2. Singh-Manoux A, Guéguen A, Martikainen P, Ferrie J, Marmot M, Shipley M. Self-rated health and mortality: short-and long-term associations in the Whitehall II study. Psychosom Med 2007;69:138-143.

3. Wennberg P, Rolandsson O, Jerdén L, Boeing H, Sluik D, Kaaks R, et al. Self-rated health and mortality in individuals with diabetes mellitus: prospective cohort study. BMJ Open 2012;2:e000760.

4. Hayes AJ, Clarke PM, Glasziou PG, Simes RJ, Drury PL, Keech AC. Can self-rated health scores be used for risk prediction in patients with type 2 diabetes? Diabetes Care 2008;31:795-7.

5. Klein BE, Klein R, Moss SE. Self-rated health and diabetes of long duration: the Wisconsin Epidemiologic Study of Diabetic Retinopathy. Diabetes Care 1998;21:236-40.

6. Pop-Busui R, Boulton AJ, Feldman EL, Bril V, Freeman R, Malik RA, et al. Diabetic Neuropathy: A Position Statement by the American Diabetes Association. Diabetes Care 2017;40:136-154.

7. Riddle MC, Herman WH. The Cost of Diabetes Care-An Elephant in the Room. Diabetes Care 2018;41: 929-32

8. Vileikyte L, Gonzalez JS. Recognition and management of psychosocial issues in diabetic neuropathy. In Handbook of clinical neurology 2014 (Vol. 126, pp. 195-209). Elsevier.

9. Vileikyte L, Leventhal H, Gonzalez JS, Peyrot M, Rubin RR, Ulbrecht JS, et al. Diabetic peripheral neuropathy and depressive symptoms: the association revisited. Diabetes Care 2005;28:2378-83.

10. Vileikyte L, Peyrot M, Gonzalez JS, Rubin RR, Garrow AP, Stickings D, et al. Predictors of depressive symptoms in persons with diabetic peripheral neuropathy: a longitudinal study. Diabetologia 2009;52:1265-73.

11. Crews RT, Shen BJ, Campbell L, Lamont PJ, Boulton AJ, Peyrot M, et al. Role and determinants of adherence to off-loading in diabetic foot ulcer healing: A Prospective Investigation. Diabetes Care 2016; 39: 1371-7.

12. Gonzalez JS, Vileikyte L, Ulbrecht JS, Rubin RR, Garrow AP, Delgado C, et al. Depression predicts first but not recurrent diabetic foot ulcers. Diabetologia 2010;53:2241-8.

13. John OP, Donahue EM, Kentle, RL. The big five inventory - versions 4a and 54. Berkeley, CA: University of California, Berkeley, Institute of Personality and Social Research, 1991.

14. Young MJ, Boulton AJ, MacLeod AF, Williams DR, Sonksen PH. A multicentre study of the prevalence of diabetic peripheral neuropathy in the United Kingdom hospital clinic population. Diabetologia 1993;36:150-4.

15. Vileikyte L, Peyrot M, Bundy C, Rubin RR, Leventhal H, Mora P, et al. The development and validation of a neuropathy-and foot ulcer-specific quality of life instrument. Diabetes Care 2003;26:2549-55.

16. Zigmond AS, Snaith RP. The hospital anxiety and depression scale. Acta Psychiatr Scand 1983;67:361-70.

17. Gandek B, Ware JE, Aaronson NK, Apolone G, Bjorner JB, Brazier JE, et al. Crossvalidation of item selection and scoring for the SF-12 Health Survey in nine countries: results from the IQOLA Project. J Clin Epidemiol 1998;51:1171-8. 
18. Nguyen CD, Carlin JB, Lee KJ. Model checking in multiple imputation: an overview and case study. Emerg Themes Epidemiol 2017;14:8-19.

19. StataCorp. Version 15.1. StataCorp College Station, TX: Released 2018

20. Dyck PJ, Davies JL, Litchy WJ, O'brien PC. Longitudinal assessment of diabetic polyneuropathy using a composite score in the Rochester Diabetic Neuropathy Study cohort. Neurology 1997;49:229-39.

21. Iversen MM, Midthjell K, Tell GS, Moum T, Østbye T, Nortvedt MW, et al. The association between history of diabetic foot ulcer, perceived health and psychological distress: the NordTrøndelag Health Study. BMC Endocr Disord 2009;9:18-25.

22. Nielsen AB, Gannik D, Siersma V, de Fine Olivarius N. The relationship between HbA1c level, symptoms and self-rated health in type 2 diabetic patients. Scand J Prim Health Care 2011;29:157-64.

23. Brown SJ, Boulton AJ, Vileikyte L, Reeves ND. Neuropathy-Related Unsteadiness and Psychosocial Outcomes in Diabetes-Preliminary Findings. Diabetes 2018; 67(Supplement 1)

24. Badawi G, Gariépy G, Pagé V, Schmitz N. Indicators of self-rated health in the Canadian population with diabetes. Diabet Med 2012;29:1021-8.

25. Molarius A, Janson S. Self-rated health, chronic diseases, and symptoms among middle-aged and elderly men and women. Journal of clinical epidemiology. 2002 Apr 1;55(4):364-70.

26. Reeves ND, Brown SJ, Petrovic M, Boulton AJ, Vileikyte L. How does self-perceived unsteadiness influence balance and gait in people with diabetes? Preliminary observations. Diabetes Care 2017;40:e51-2.

27. Brown SJ, Boulton AJ, Vileikyte L, Reeves ND. Neuropathy-Related Unsteadiness and Psychosocial Outcomes in Diabetes_-Preliminary Findings. Diabetes 2018;67 (supplement 1)

28. Bradley C. Importance of differentiating health status from quality of life. Lancet 2001;357:7-8.

29. Hoogendoorn CJ, Shapira A, Roy J, Kane NS, Gonzalez JS. Diabetes distress and quality of life in adults with diabetes. In: Delamater AM, Marrero DG, eds. Behavioral Diabetes. New York: Springer, 2020; 303-328).

30. American Diabetes Association. Lifestyle management: standards of medical care in diabetes- 2019. Diabetes Care 2019;41: S38-S50. 


\begin{tabular}{|c|c|c|c|}
\hline Characteristics & UK $(n=179)$ & USA $(n=116)$ & Total $(n=295)$ \\
\hline Female Gender & $29.1 \%$ & $32.8 \%$ & $30.5 \%$ \\
\hline Age & $61.2 \pm 11.3$ & $61.9 \pm 9.7$ & $61.5 \pm 10.7$ \\
\hline $\begin{array}{l}\text { Education: } \\
\text { Primary } \\
\text { Secondary } \\
\text { Some College } \\
\text { College Grad } \\
\text { Postgraduate }\end{array}$ & $\begin{array}{c}4.0 \% \\
54.3 \% \\
28.0 \% \\
7.4 \% \\
6.3 \%\end{array}$ & $\begin{array}{c}0.9 \% \\
42.5 \% \\
14.2 \% \\
26.5 \% \\
15.9 \%\end{array}$ & $\begin{array}{c}2.8 \% \\
49.7 \% \\
22.6 \% \\
14.9 \% \\
10.1 \%\end{array}$ \\
\hline Marital Status (living alone) & $31.8 \%$ & $25.0 \%$ & $29.2 \%$ \\
\hline Diabetes Type (type 2) & $65.9 \%$ & $82.8 \%$ & $72.7 \%$ \\
\hline Retinopathy & $45.0 \%$ & $42.6 \%$ & $44.1 \%$ \\
\hline Nephropathy & $14.0 \%$ & $16.4 \%$ & $14.9 \%$ \\
\hline Cardiovascular Disease & $31.3 \%$ & $40.5 \%$ & $34.9 \%$ \\
\hline Concomitant Disorders (\#) & $0.7 \pm 0.9$ & $1.5 \pm 1.3$ & $1.0 \pm 1.1$ \\
\hline $\begin{array}{l}\text { DPN Severity: } \\
\text { Neuropathy Disability Score } \\
\text { Vibration Perception Threshold }\end{array}$ & $\begin{array}{l}7.3 \pm 2.3 \\
39.3 \pm 9.5\end{array}$ & $\begin{array}{c}7.5 \pm 2.2 \\
45.4 \pm 8.4\end{array}$ & $\begin{array}{c}7.4 \pm 2.3 \\
41.8 \pm 9.5\end{array}$ \\
\hline $\begin{array}{l}\text { Foot Ulcer: } \\
\text { Ever/Current }\end{array}$ & $30.2 \%$ & $50.9 \%$ & $38.3 \%$ \\
\hline Neuroticism & $2.7 \pm 0.7$ & $2.7 \pm 0.6$ & $2.7 \pm 0.7$ \\
\hline NeuroQoL - Pain & $1.9 \pm 0.8$ & $1.9 \pm 0.7$ & $1.9 \pm 0.8$ \\
\hline NeuroQoL - Reduced Feeling & $2.6 \pm 1.5$ & $3.5 \pm 1.3$ & $2.9 \pm 1.5$ \\
\hline NeuroQoL - Unsteadiness & $2.1 \pm 1.2$ & $2.4 \pm 1.1$ & $2.2 \pm 1.1$ \\
\hline NeuroQoL - Limitations daily activities & $2.3 \pm 1.3$ & $2.9 \pm 1.3$ & $2.5 \pm 1.4$ \\
\hline HADS Depression & $5.1 \pm 4.1$ & $4.7 \pm 3.4$ & $4.9 \pm 3.8$ \\
\hline Self-Rated Health & $3.3 \pm 1.1$ & $3.2 \pm 0.9$ & $3.2 \pm 1.0$ \\
\hline
\end{tabular}

Note: Values are percentages and means \pm standard deviations.

Table 1. Sample Characteristics 


\begin{tabular}{|c|c|c|c|c|c|c|}
\hline Variables & $\begin{array}{c}\text { Model 1 } \\
\text { Coef.(95\%CI) } \\
\end{array}$ & $\begin{array}{c}\text { Model 2 } \\
\text { Coef.(95\% CI) } \\
\end{array}$ & $\begin{array}{c}\text { Model 3 } \\
\text { Coef. }(95 \% \mathrm{CI}) \\
\end{array}$ & $\begin{array}{c}\text { Model } 4 \\
\text { Coef.(95\%CI) } \\
\end{array}$ & $\begin{array}{c}\text { Model 5 } \\
\text { Coef. }(95 \% \mathrm{CI}) \\
\end{array}$ & $\begin{array}{c}\text { Model 6 } \\
\text { Coef. }(95 \% \mathrm{CI}) \\
\end{array}$ \\
\hline \multicolumn{7}{|l|}{ Demo/Disease/Trait } \\
\hline Country & $.27(.04-.50)$ & $.24(.01-.48)$ & $.25(.01-.49)$ & $.31(.08-.54)$ & $.34(.11-.57)$ & $.23(.01-.46)$ \\
\hline Female Sex & $-.09(-.32-.15)$ & $-.08(-.32-.15)$ & $-.08(-.32-.16)$ & $-.01(-.24-.22)$ & $.004(-.22-.23)$ & $.02(-.19-.24)$ \\
\hline Type 2 Diabetes & $-.28(-.56-.01)$ & $-.30(-.58--.02)$ & $-.30(-.58--.02)$ & $-.20(-.47-.07)$ & $-.20(-.46-.07)$ & $-.17(-.42-.09)$ \\
\hline Age & $.02(.01-.03)$ & $.02(.01-.03)$ & $.02(.01-.03)$ & $.02(.004-.03)$ & $.01(.003-.03)$ & $.01(.003-.03)$ \\
\hline Retinopathy & $-.18(-.41-.05)$ & $-.15(-.38-.08)$ & $-.14(-.38-.09)$ & $-.10(-.32-.12)$ & $-.10(-.32-.12)$ & $-.09(-.30-.11)$ \\
\hline \multicolumn{7}{|l|}{ Nephropathy } \\
\hline Some & $-.29(-.59-.01)$ & $-.28(-.58-.02)$ & $-.27(-.58-.03)$ & $-.23(-.51-.06)$ & $-.20(-.49-.08)$ & $-.18(-.45-.09)$ \\
\hline Dialysis & $-.38(-1.4-.68)$ & $-.31(-1.4-.75)$ & $-.30(-1.4-.75)$ & $-.34(-1.3-.66)$ & $-.31(-1.3-.68)$ & $-.38(-1.3-.57)$ \\
\hline CV Disease & $-.38(-.61--.15)$ & $-.39(-.62--.17)$ & $-.39(-.62--.17)$ & $-.33(-.55-. .12)$ & $-.31(-.53--.10)$ & $-.31(-.52--.11)$ \\
\hline Comorbidities & $-.12(-.22--.01)$ & $-.11(-.22--.01)$ & $-.11(-.22--.01)$ & $-.08(-.18-.02)$ & $-.09(-.19-. .01)$ & $-.09(-.19-.001)$ \\
\hline Neuroticism & $-.49(-.65--.32)$ & $-.49(-.65-. .33)$ & $-.49(-.65-. .33)$ & $-.34(-.51--.18)$ & $-.32(-.48--.15)$ & $-.17(-.34--.001)$ \\
\hline \multicolumn{7}{|l|}{ DPN Severity } \\
\hline NDS & & $-.06(-.11--.01)$ & $-.06(-.11--.01)$ & $-.01(-.05-.06)$ & $.002(-.05-.06)$ & $-.01(-.04-.06)$ \\
\hline VPT & & $.01(-.01-.02)$ & $.01(-.01-.02)$ & $.01(-.002-.02)$ & $.01(-.001-.02)$ & $.01(.002-.03)$ \\
\hline DFU History & & & $-.05(-.29-.20)$ & $-.03(-.26-.21)$ & $.04(-.20-.27)$ & $.002(-.23-.24)$ \\
\hline \multicolumn{7}{|l|}{ DPN Symptoms } \\
\hline Reduced Feeling & & & & $-.11(-.20-. .01)$ & $-.08(-.18-.02)$ & $-.06(-.15-.04)$ \\
\hline Pain & & & & $-.17(-.31--.02)$ & $-.13(-.28-.03)$ & $-.01(-.17-.14)$ \\
\hline Unsteadiness & & & & $-.16(-.28--.04)$ & $-.13(-.25-. .01)$ & $-.07(-.19-.05)$ \\
\hline $\begin{array}{l}\text { DPN-specific Limitations in } \\
\text { Daily Activities }\end{array}$ & & & & & $-.13(-.22--.04)$ & $-.09(-.18-.01)$ \\
\hline \multicolumn{7}{|l|}{ Emotional Distress } \\
\hline Depressive Sx & & & & & & $-.09(-.12--.05)$ \\
\hline DPN-specific distress & & & & & & $-.03(-.17-.12)$ \\
\hline Joint Significance Test & $3.5(2.6-4.3)$ & $3.6(2.7-4.6)$ & $3.6(2.7-4.6)$ & $3.6(2.6-4.5)$ & $3.6(2.7-4.6)$ & $3.1(2.2-4.0)$ \\
\hline
\end{tabular}

Table 2. Regression Models Predicting SRH at Baseline 


\begin{tabular}{lc}
\hline & Model 7 \\
Variables & Coef.(95\%CI) \\
Demo/Disease/Trait & \\
Time & $-.06(-.15-.03)$ \\
Country & $.22(.04-.41)$ \\
Female Sex & $-.13(-.31-.06)$ \\
Type 2 Diabetes & $-.18(-.40-.04)$ \\
Age & $.01(-.00-.02)$ \\
Retinopathy & $.02(-.16-.12)$ \\
Nephropathy & \\
Some & $-.19(-.38-.01)$ \\
Dialysis & $-.29(-.91-.33)$ \\
NDS & $.02(-.01-.05)$ \\
DPN Symptoms & \\
Reduced Feeling & $-.06(-.15-.04)$ \\
Pain & $-.01(-.17-.14)$ \\
Unsteadiness & $-.07(-.19--.01)$ \\
DPN-Specific Limitations in & $-.08(-.14--.02)$ \\
Daily Activities & \\
Emotional Distress & \\
Depressive Sx & $-.06(-.09--.04)$ \\
DPN-specific distress & $-.10(-.20--.001)$ \\
Joint Significance Test & \\
\hline
\end{tabular}

Table 3. Mixed-effects Model Predicting SRH at 18 months 